\title{
Modelling of geomagnetic induction in pipelines
}

\author{
L. Trichtchenko and D. H. Boteler \\ Geomagnetic Laboratory, Geological Survey of Canada, 7 Observatory Crescent, Ottawa, Ontario K1A 0Y3, Canada
}

Received: 9 October 2001 - Revised: 17 January 2002 - Accepted: 23 January 2002

\begin{abstract}
Geomagnetic field variations induce telluric currents in pipelines, which modify the electrochemical conditions at the pipe/soil interface, possibly contributing to corrosion of the pipeline steel. Modelling of geomagnetic induction in pipelines can be accomplished by combining several techniques. Starting with geomagnetic field data, the geoelectric fields in the absence of the pipeline were calculated using the surface impedance derived from a layeredEarth conductivity model. The influence of the pipeline on the electric fields was then examined using an infinitely long cylinder (ILC) model. Pipe-to-soil potentials produced by the electric field induced in the pipeline were calculated using a distributed source transmission line (DSTL) model. The geomagnetic induction process is frequency dependent; therefore, the calculations are best performed in the frequency domain, using a Fourier transform to go from the original time domain magnetic data, and an inverse Fourier transform at the end of the process, to obtain the pipe-tosoil potential variation in the time domain. Examples of the model calculations are presented and compared to observations made on a long pipeline in the auroral zone.
\end{abstract}

Key words. Geomagnetism and paleomagnetism (geomagnetic induction)

\section{Introduction}

Space weather produces geomagnetic disturbances that affect a variety of technological systems on the ground (Lanzerotti and Gregori, 1986; Boteler et al., 1998). In pipelines the geomagnetic disturbances induce large electric currents (Campbell, 1978; 1980) and changes in the potential of the pipeline with respect to the surrounding soil (Shapka, 1993). The potential difference between a pipeline and the adjacent Earth strongly influences the electrochemical environment at the pipeline surface, which influences possible corrosion occur-

Correspondence to: L. Trichtchenko

(ltrichtc@NRCan.gc.ca) rence. Because of this, sacrificial anodes and rectifier units are connected to the pipeline steel to drive it negatively with respect to the surrounding soil. In this process the pipeline becomes the cathode of the circuit, leading to the name "cathodic protection" (Peabody, 2000). Fluctuations in pipe-tosoil potential difference, produced by geomagnetic disturbances and other causes, change the electrochemical conditions of the pipe, meaning that it is temporarily not protected. There is concern that, over time, this intermittent loss of cathodic protection may contribute to corrosion of the pipeline. The pipe-to-soil potential (PSP) fluctuations also interfere with pipeline surveys, which are made to check the integrity of the cathodic protection on the pipeline (Place and Sneath, 2001).

For designing cathodic protection for new pipelines and for understanding protection surveys on existing pipelines, it is useful to be able to determine the potential variations that can be expected at different places on a pipeline during geomagnetic disturbances. Figure 1 shows a block diagram of the steps involved in modelling these effects of geomagnetic disturbances on pipelines. Recordings of the geomagnetic variations in the region of the pipeline can be obtained from permanent magnetic observatories or specially installed magnetometers. A forward Fourier transform of the magnetic recordings is used to give the amplitude spectrum of the geomagnetic variations. Multiplying this by the surface impedance, derived from a multi-layer Earth conductivity model, gives the amplitude spectrum of the electric field at the Earth's surface. An infinitely long cylinder (ILC) model of the pipeline is then used to determine how the presence of the pipeline modifies the electric fields in the nearby soil and in the pipe itself. The induced electric field in the pipeline is used as input for a distributed source transmission line (DSTL) model of the pipeline to give the pipe-to-soil potential as a function of distance along the pipeline. Finally, an inverse Fourier transform converts these frequency domain results into the time variations of the pipe-to-soil potential at any location on the pipeline.

In this paper, we describe each step of the modelling pro- 
TIME DOMAIN

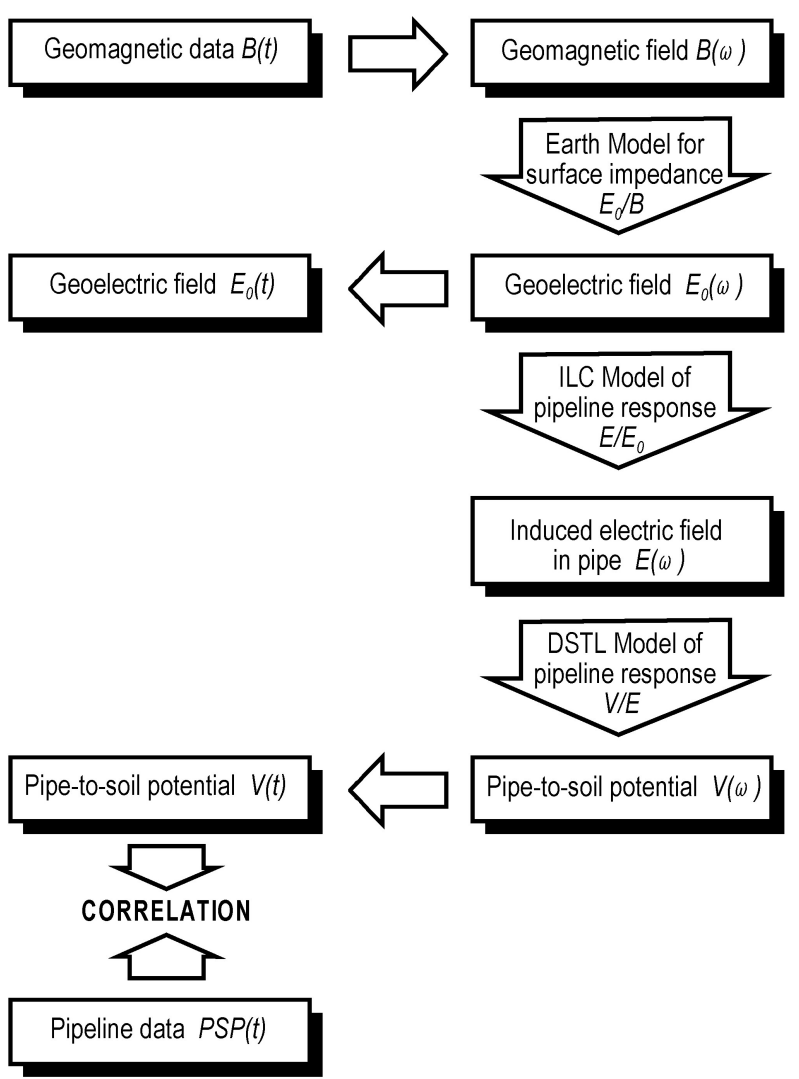

Fig. 1. The process of calculating a pipeline response to geomagnetic disturbances.

cess. Then, using magnetic data from a nearby recording site as an input to the model, calculations of the pipe-tosoil potential variations produced on a real pipeline in the auroral zone were made. The pipeline is $868 \mathrm{~km}$ long and extends from Norman Wells to Zama in northern Canada, spanning geomagnetic latitudes from $65^{\circ} \mathrm{N}$ to $70^{\circ} \mathrm{N}$. Measurements of the pipe-to-soil potential variations were done in June 1998 as part of an international study of telluric effects on pipelines (Boteler and Trichtchenko, 2000). A comparison is made between the model results and the observed pipe-to-soil potentials.

\section{Electric fields at the Earth's surface}

The electric fields produced by geomagnetic disturbances drive electric currents within the Earth. These induced currents have the effect of shielding the interior of the Earth from the geomagnetic disturbance. The fall-off of the magnetic and electric fields within the Earth is dependent on frequency and on the conductivity structure of the Earth. At the frequencies of geomagnetic field variations, the skin depths within the Earth extend to hundreds of kilometers, and the conductivity of the Earth down to these depths has to be taken into account in calculating the relation between the electric and magnetic fields at the surface.

The variation of conductivity with depth within the Earth can be modelled using multiple horizontal layers with a different uniform conductivity, as shown in Fig. 2, with the last layer as a uniform half-space. For the calculation of the geoelectric field, an assumption also needs to be made about the spatial structure of the source of geomagnetic fluctuations. The period studied (June 1998) was geomagnetically quiet; therefore, we assumed the simplest case of a plane wave, propagating down into the Earth.

We use the geomagnetic coordinate system with axis $x$ north, $y$ east, and $z$ vertically downwards. For the frequency range of $1 \mathrm{~s}$ to $24 \mathrm{~h}$ and Earth resistivities $1-1000 \mathrm{Ohm}-\mathrm{m}$, displacement currents are small in comparison with conductivity currents. Therefore, electric and magnetic fields in the frequency domain can be given by the diffusion equations

$$
\begin{aligned}
& \frac{d^{2} E}{d z^{2}}=i \omega \mu \sigma E, \\
& \frac{d^{2} H}{d z^{2}}=i \omega \mu \sigma H .
\end{aligned}
$$

Solutions for each layer have the form

$$
E=A\left(e^{-k z}+R e^{k z}\right)
$$

and

$$
H=A\left(\frac{e^{-k z}}{Z_{0}}-R \frac{e^{k z}}{Z_{0}}\right)
$$

where $A$ and $R$ are the amplitude and reflection coefficients $k=\sqrt{i \omega \mu \sigma}$ is the propagation constant, $Z_{0}=i \omega \mu / k=$ $\sqrt{i \omega \mu / \sigma}$ is the characteristic impedance (ratio of the electric and magnetic fields for the uniform medium).

For our case, when the magnetic field at the surface of the Earth (first layer) is known from the magnetic observations, the electric field can be obtained from the ratio (impedance) of magnetic and electric fields

$E_{\text {surface }}=Z_{1} H_{\text {surface }}$.

The impedance at any layer $n$ can be found by applying the recursion relation for the impedance of an $N$-layered halfspace (Weaver, 1994, p.293).

$Z_{n}=i \omega \mu\left(\frac{1-r_{n} e^{-2 k_{n} d_{n}}}{k_{n}\left(1+r_{n} e^{-2 k_{n} d_{n}}\right)}\right)$,

where $d_{n}, k_{n}$ are the thickness and propagation constants of the layer $n$,

$r_{n}=\frac{1-k_{n} \frac{Z_{n+1}}{i \omega \mu}}{1+k_{n} \frac{Z_{n+1}}{i \omega \mu}}$

and for the last layer $Z_{n}=i \omega \mu / k_{N}$.

The electric field in the frequency domain was calculated using the layered Earth conductivity model of Ferguson and Odwar (1997) for the northwestern region of Canada where 


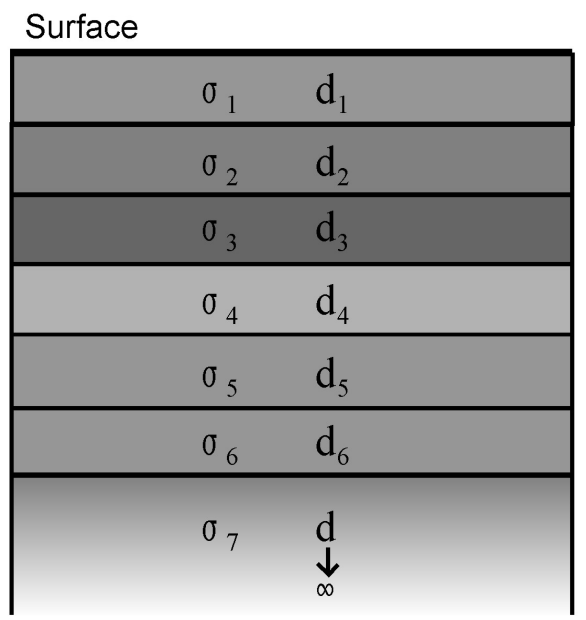

Fig. 2. Layered conductivity model for the Earth beneath the Norman Wells - Zama pipeline.

the modelled pipeline is located. The Earth model comprises 7 layers with thicknesses (d) of $0.025,6,15,25,60,300 \mathrm{~km}$ and an underlying half-space and resistivities $(1 / \sigma)$ of 50 , 20, 5000, 300, 3000, 300 and 10 Ohm-m, as shown in Fig. 2. The variation of the surface impedance with frequency for this layered Earth conductivity model is shown in Fig. 3.

\section{Electric fields inside a long pipeline}

In the above section, we have considered the generation of electric fields at the Earth's surface in the absence of a pipeline. Where there is a pipeline, reflections from the pipe modify the electric and magnetic fields in the surrounding soil and this also means that the electric field inside the pipe is not necessarily the same as the electric field away from the pipe. To determine the relationship between the electric field in the pipe steel and the incident electric field in the Earth, calculated in the previous section, a pipeline can be modelled as an infinitely long cylinder (ILC).

The mathematical basis for this model is given by Kaufman and Keller (1981). The multilayered cylinder is aligned with the $y$-axis and is placed in the conducting Earth (layer 1). To apply this model to pipelines we use a cylinder with three layers: the outside insulating coating (layer 2), the pipe steel (layer 3), and the gas/oil inside the pipe (layer 4), each with its corresponding conductivity and permeability, as shown in Fig. 4.

For an electromagnetic field with $E$ parallel to the cylinder, the electric field in any layer satisfies the diffusion equation in cylindrical coordinates

$$
\frac{\partial^{2} E}{\partial r^{2}}+\frac{1}{r} \frac{\partial E}{\partial r}+\frac{1}{r^{2}} \frac{\partial^{2} E}{\partial \phi^{2}}-k^{2} E=0
$$

where $k$ is the propagation constant of the particular layer given by $k=\sqrt{i \omega \mu \sigma}$. The electric field in any layer except
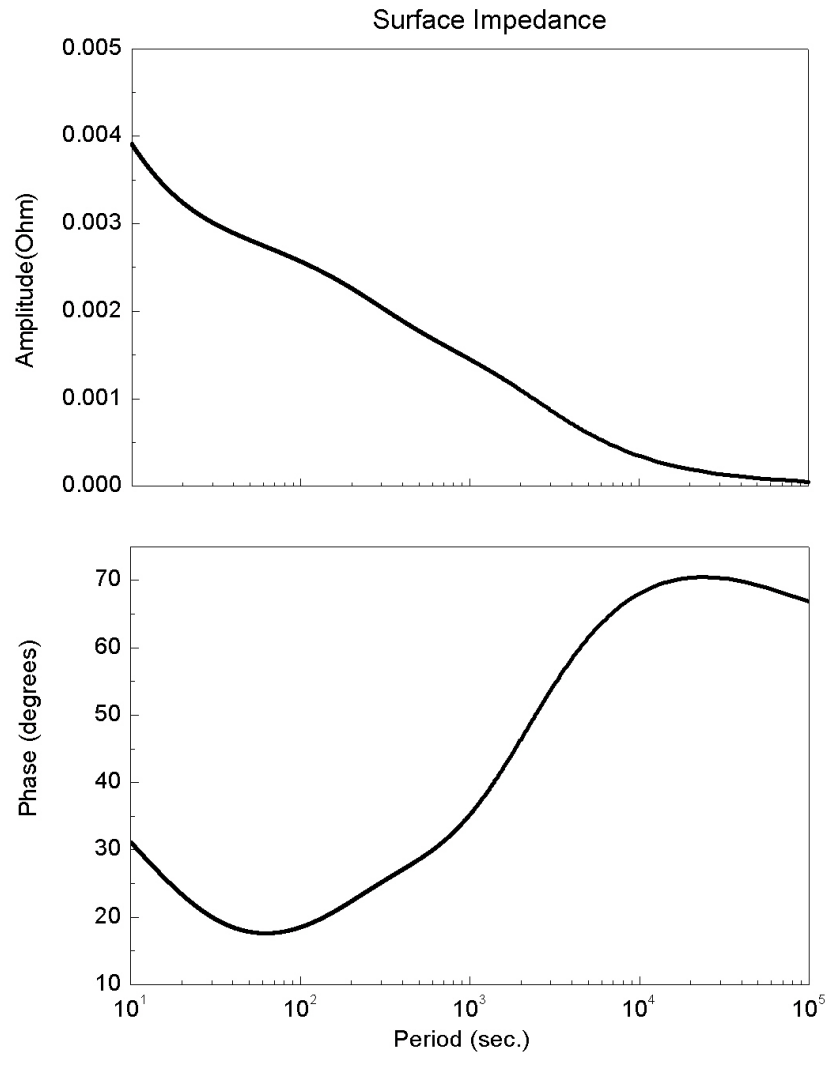

Fig. 3. Surface impedance for the layered Earth model shown in Fig. 2.

the inner can be represented as the sum of incident and reflected parts. For the innermost layer, only the incident part exists. The electric field in the pipeline steel $E_{p}$ is given by

$E_{p}=\sum_{n=0}^{\infty} A_{p n}\left[I_{n}\left(k_{p} r\right)+R_{p n} K_{n}\left(k_{p} r\right)\right] \cos n \phi$,

where $k_{p}$ is the propagation constant in pipeline steel, $I_{n}\left(k_{p} r\right), K_{n}\left(k_{p} r\right)$ are modified Bessel functions of the first and second kinds. In this equation the first term represents the incident wave coming in from the outer layer and the second term represents the wave reflected from the boundary with the inner layer.

Corresponding expressions can be written for the other layers, i.e. the surrounding Earth, the pipe coating and the oil or gas in the pipe (Trichtchenko and Boteler, 2001). In these expressions, the amplitudes $A_{n}$ and reflection coefficients $R_{n}$ for each layer can be found from the boundary conditions and are defined in terms of the amplitude of the electric field in the Earth, described in the previous section, as well as the conductivity $\sigma$ and magnetic permeability $\mu$ of each layer. These boundary conditions are the conservation of the electric field at each boundary with known (zero) reflection coefficient for the innermost layer and known incident electric field at the outer boundary (Earth electric field).

For the present study we have set up an ILC model for the electromagnetic characteristics of the Norman Wells - 


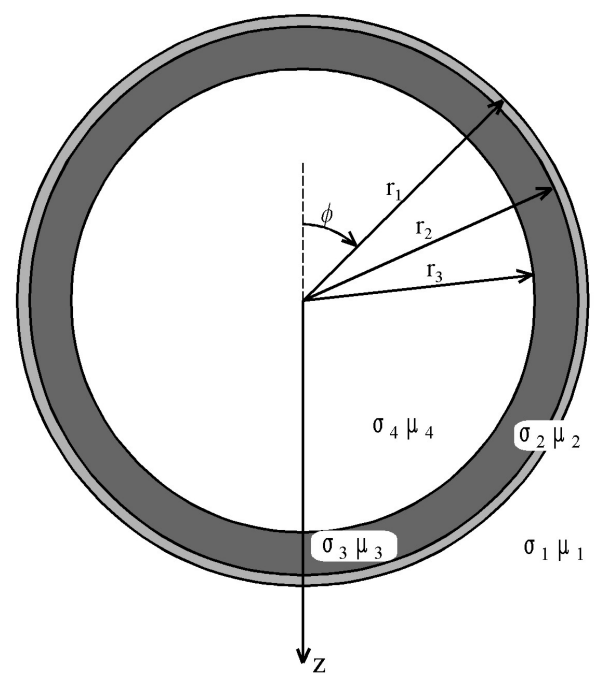

Fig. 4. Coordinate system and characteristics of the pipeline layers.

Zama oil pipeline (Boteler and Trichtchenko, 2000: vol 2, Appendix C). The diameter of the pipeline is $32.4 \mathrm{~cm}$ with $7 \mathrm{~mm}$ wall thickness. The pipeline steel has a conductivity of $5.5 \cdot 10^{6} \mathrm{~S} / \mathrm{m}$. The pipeline coating is $0.7 \mathrm{~mm}$ thick and is assumed to have a conductivity of $10^{-6} \mathrm{~S} / \mathrm{m}$. For the oil inside the pipeline we use a conductivity value of $1.0 \mathrm{~S} / \mathrm{m}$, and for the soil, $0.02 \mathrm{~S} / \mathrm{m}$. The soil, the pipeline coating and the oil are assumed to have the free space value $\left(4 \pi \cdot 10^{-7}\right)$ for the magnetic permeability and for the pipeline steel the relative magnetic permeability equals 180 (Weast, 1974).

Figure 5 shows the ratio between the electric field in the pipe steel and the electric field in the ground (in the absence of the pipe) as a function of frequency. This shows that the electric field is the same as the electric field in the ground for periods above approximately $10 \mathrm{~s}$. At periods less than $10 \mathrm{~s}$, the electric field in the pipe is increasingly attenuated as the frequency increases.

\section{Modelling pipe-to-soil potentials}

The one-dimensional (ILC) approach to the pipeline section, used in Sect. 3, is restricted to the calculations only with the electric fields in the uniform pipeline far from the discontinuities, such as bends, branches and ends of the pipeline network. The effect of electric fields induced in real pipelines or pipeline networks, including the discontinuities mentioned, can be modelled by distributed-source transmission line (DSTL) theory. This has been used extensively for AC induction in pipelines (Taflove and Dabkowski, 1979) and applied to geomagnetic induction in pipelines by Boteler and Seager (1998). In the DSTL approach, each uniform section of the pipeline is represented by a transmission line circuit element with specific series impedance and a parallel admittance. The induced electric field is represented by voltage sources distributed along the transmission line (Fig. 6).
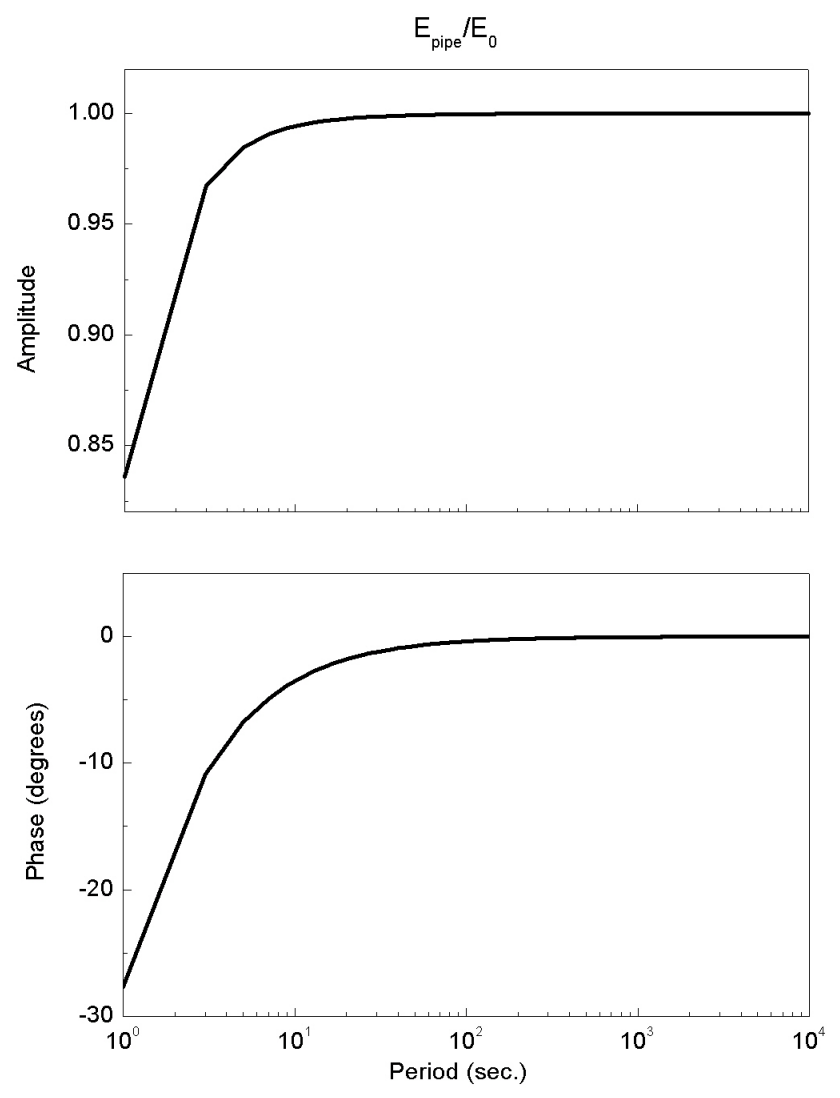

Fig. 5. Ratio between the electric field in the pipe steel and the electric field in the ground for the Norman Wells - Zama pipeline.

The basic equations describing the voltage and current produced in any section of pipeline by an induced electric field $E_{p}$ are

$$
\begin{gathered}
\frac{d^{2} V_{p}}{d x^{2}}-\gamma^{2} V_{p}=\frac{d E_{p}}{d x}, \\
\frac{d^{2} I_{p}}{d x^{2}}-\gamma^{2} I_{p}=-Y E_{p},
\end{gathered}
$$

where $\gamma$ is the propagation constant along the pipeline, defined as $\gamma=\sqrt{Z Y}, Y=G+i \omega C$ is the parallel admittance and $Z=R+i \omega L$ is series impedance per unit length with $G=$ conductance to ground, $C=$ capacitance, $R=$ resistance of pipeline steel, $L=$ inductance. From the ILC model it follows that pipeline response at periods larger than $10 \mathrm{~s}$ is independent of frequency (Fig. 5). For that reason, frequencydependent parts, such as capacitance $C$ and inductance $L$, were not included in the modelling.

Equations (10) and (11) for the section of the pipeline with a uniform induced electric field have the solutions of the form (Boteler and Seager, 1998)

$$
\begin{aligned}
V_{p} & =\frac{E_{p}}{\gamma}\left(A_{p} e^{-\gamma\left(x-x_{1}\right)}-B_{p} e^{-\gamma\left(x_{2}-x\right)}\right), \\
I_{p} & =\frac{E_{p}}{\gamma Z_{c}}\left(1+A_{p} e^{-\gamma\left(x-x_{1}\right)}+B_{p} e^{-\gamma\left(x_{2}-x\right)}\right),
\end{aligned}
$$




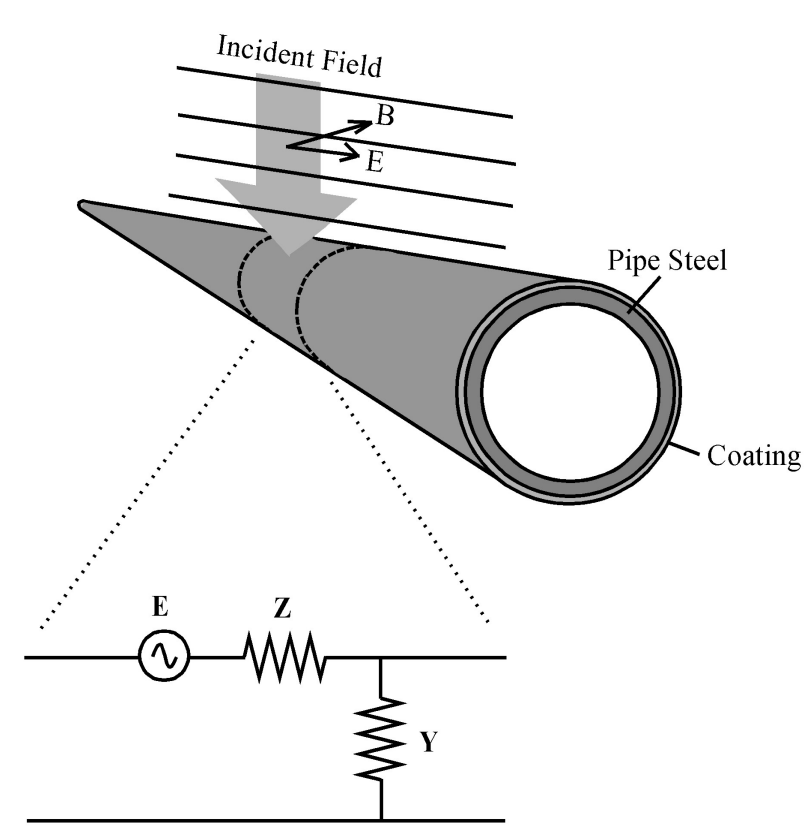

Fig. 6. Transmission line model of pipeline including distributed voltage sources representing the induced electric field.

where $x_{1}$ and $x_{2}$ are the positions of the ends of the pipeline, $A_{p}$ and $B_{p}$ are constants dependent on the boundary conditions at the ends of the pipeline, $Z_{c}=\sqrt{Z / Y}$ is the characteristic impedance of the pipeline.

The termination at the end of any pipeline section may be other sections of pipe, perhaps with different electrical properties, each experiencing the induced electric field. To model this we use Thevenin's theorem that the effect of any circuit can be represented by an equivalent circuit comprising a voltage source in a series with impedance. Calculations start from the last section of the pipeline using the known resistance to ground and the electric field in it. The values of the components of the Thevenin equivalent circuit can be consequently calculated by considering the open-circuit voltage and short-circuit impedance of each section, as shown by Boteler (1997).

Circuit characteristics for the DSTL modelling of the Norman Wells - Zama pipeline (Fig. 7) are shown in Table 1. These values were used with Eq. (12) to calculate the pipeto-soil potentials produced by northward and eastward electric fields (Fig. 8). The results show the characteristic features that the largest pipe-to-soil potentials are produced at discontinuities in the pipeline, such as bends or the ends of the pipeline. Away from these peak values, the pipe-to-soil potentials have an exponential fall-off with distance characterized by adjustment distance $1 / \gamma$. The resistance to ground (terminating impedances) at the ends of the pipeline has a significant effect on the end pipe-to-soil potentials. To illustrate this, Fig. 8 shows the modelling results obtained for two limiting cases: with no ground connection and with a low resistance $(0.1 \mathrm{Ohm})$ connection to ground.

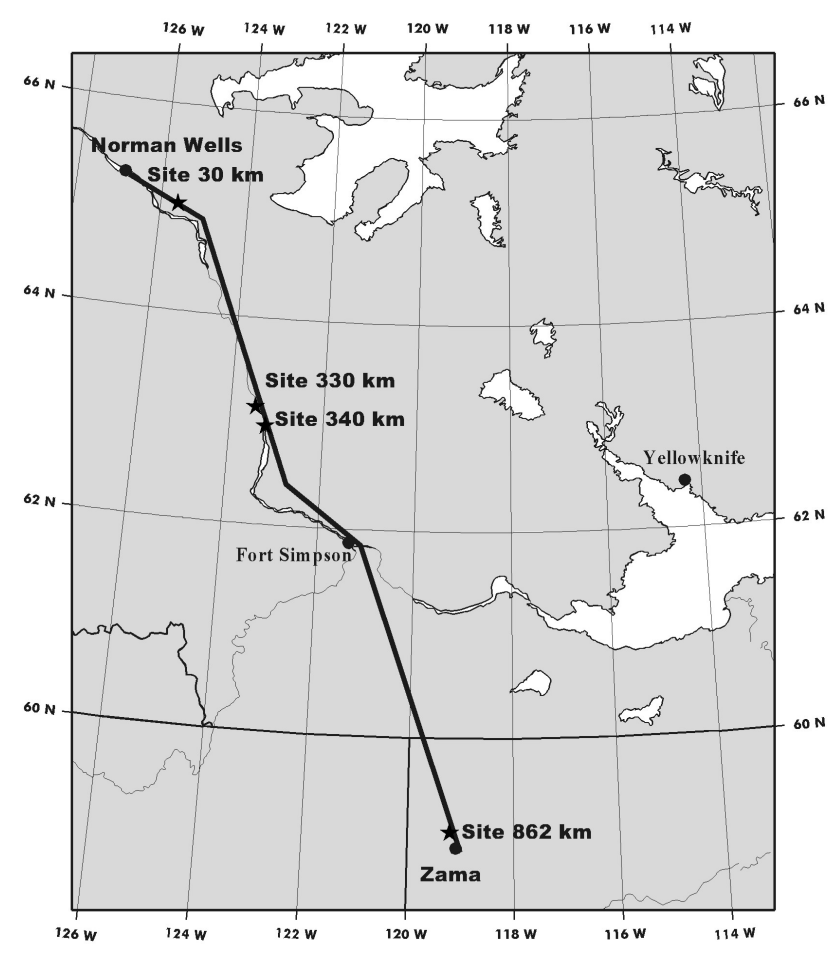

Fig. 7. Recording sites on the Norman Wells - Zama pipeline (stars), Fort Simpson magnetic observation site and Yellowknife magnetic observatory.

Table 1. Electrical characteristics of the Norman Wells to Zama Pipeline

\begin{tabular}{lc}
\hline coating resistance & $10^{5} \Omega \mathrm{m}^{2}$ \\
coating conductance & $10 \mu \mathrm{S} \mathrm{m}^{-2}$ \\
series resistance & $0.028 \Omega / \mathrm{km}$ \\
parallel admittance & $0.01 \mathrm{~S} / \mathrm{km}$ \\
characteristic impedance & $1.67 \Omega$ \\
propagation constant & $0.0167 \mathrm{~km}^{-1}$ \\
adjustment distance & $60 \mathrm{~km}$ \\
\hline
\end{tabular}

\section{Modelling results}

To give an example of the above calculations we present the results of modelling geomagnetic induction in the Norman Wells - Zama pipeline for 28 June 1998. On this date only, the magnetic data were available from the CANOPUS site at Fort Simpson near the pipeline route, and recordings were also being made of pipe-to-soil potentials. Figure 9 shows examples of the modelling process. The variations of the north magnetic field component $B_{x}=\mu_{0} H x$, recorded at Fort Simpson are shown in Fig. 9a. A discrete Fourier transform the series of $N$ data points of magnetic field horizontal components, $H_{x, y}\left(t_{k}\right)$, gives the amplitude spectrum of the 

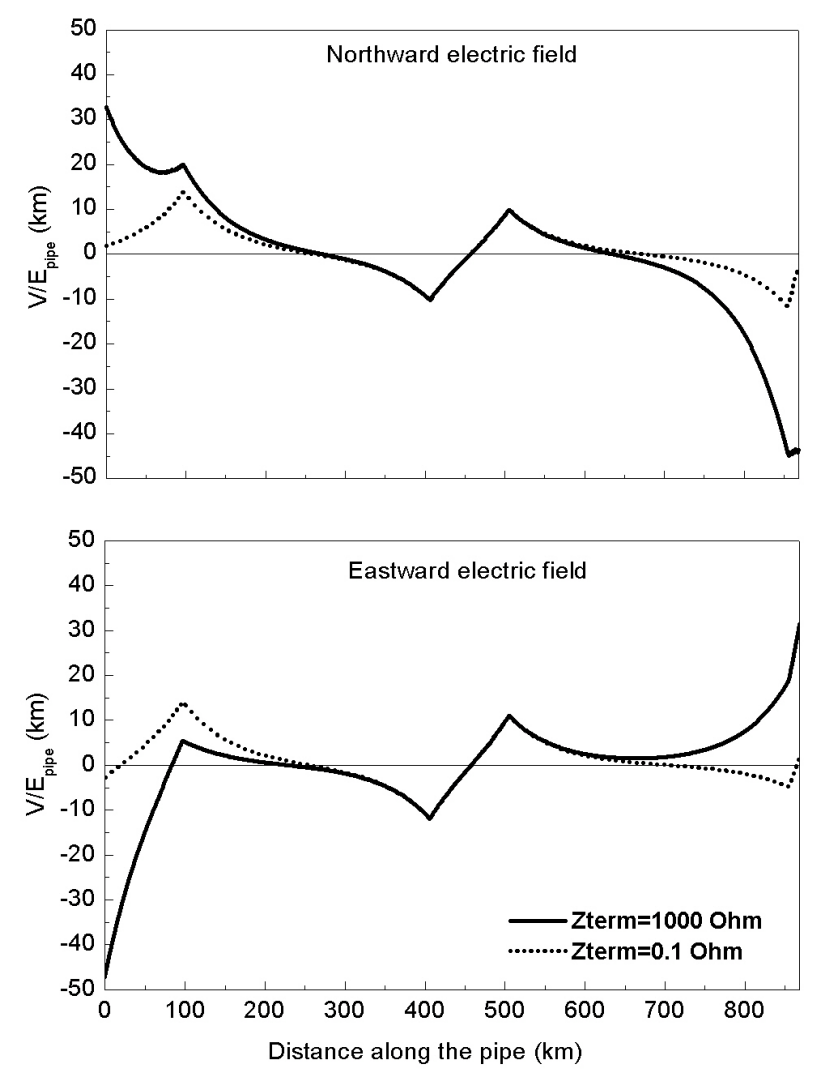

Fig. 8. Ratio of pipe-to-soil potential to the pipeline electric field based on DSTL model for Norman Wells - Zama pipeline. Solid line represents high terminating impedance $(1000 \mathrm{Ohm})$, dotted line - low terminated impedance $(0.1 \mathrm{Ohm})$.

magnetic field horizontal components variations

$H_{x, y}\left(\omega_{n}\right)=\sum_{k=0}^{N-1} H_{x, y}\left(t_{k}\right) \exp \left(i \omega_{n} t_{k}\right)$.

The variations of the amplitude spectrum for the north component of magnetic field $B_{x}$ in frequency domain is shown in Fig. 9b. From the Fort Simpson daily magnetic data with a 5 -s sampling interval, we obtain a frequency spectrum corresponding to periods of $10 \mathrm{~s}$ to $24 \mathrm{~h}$. Multiplying the spectrum of the magnetic field components by the surface impedance (Sect. 2)

$E_{x}\left(\omega_{n}\right)=Z\left(\omega_{n}\right) H_{y}\left(\omega_{n}\right)$

$E_{y}\left(\omega_{n}\right)=-Z\left(\omega_{n}\right) H_{x}\left(\omega_{n}\right)$

gives the spectra of the electric field at the ground, as shown for the $E_{y}$ component in Fig. 9d. An inverse discrete Fourier transform can then be used to define the electric field variations in the time domain (Fig. 9c).

The ILC modelling shows that the effect of reflections from the pipe only starts to become significant at periods less than $10 \mathrm{~s}$ (see Fig. 5). Therefore, for the frequency range considered here, the modelling can be done using the simplification that the electric field inside the pipeline steel is the
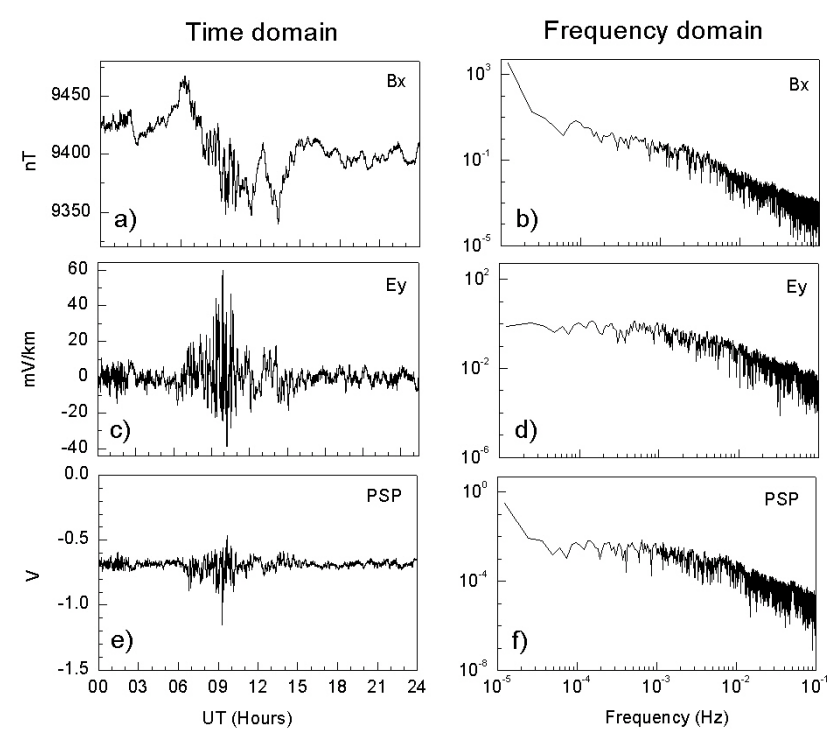

Fig. 9. Modelling results of pipeline response in time-domain and frequency domain. (a) and (b) north-south component of magnetic field (c) and (d) east-west component of the geoelectric field (e) and (f) pipe-to-soil potential at site 340 on Norman Wells - Zama pipeline.

same as the electric field at the Earth's surface in the absence of the pipeline, i.e.

$E_{\text {pipe }}=E_{\text {surface }}$

and the electric field spectrum ( $Y$-component is shown in Fig. 9d) can be used for the DSTL modelling with the resistive input values for the pipeline parameters.

To determine the frequency dependent pipe-to-soil potentials, the modelling results of Sect. 4 (Fig. 8) were combined with the electric field spectrum. In the present case, the pipeline DSTL model has only ohmic resistances, therefore, the frequency content of the modelled voltage variations only comes from the electric field. For a specified location on the pipeline $l$, Fig. 8 gives the pipeline voltage variations produced by the northward and eastward components of the electric field. These model voltage/electric field ratios, $P_{x}(l)$ and $P_{y}(l)$, are combined with the northward and eastward electric field spectra to give the spectrum of the voltage variations at the specified location

$V_{p}\left(l, \omega_{n}\right)=P_{x}(l) E_{x}\left(\omega_{n}\right)+P_{y}(l) E_{y}\left(\omega_{n}\right)$.

An example for site 340, near Fort Simpson, is shown in Fig. 9f. An inverse discrete Fourier transform of the pipeline voltage spectrum $V_{p}\left(l, \omega_{n}\right)$ is then used to obtain the pipeline voltage variations in the time domain (Fig. 9e)

$V_{p}\left(l, t_{k}\right)=\frac{1}{N} \sum_{n=0}^{N-1} V_{p}\left(l, \omega_{n}\right) \exp \left(-i \omega_{n} t_{k}\right)$.

Model calculations were made for four sites on the Norman Wells - Zama pipeline: one near each end and two sites about one-third of the way along the pipeline. The site numbers refer to the distance, in kilometers, from the northern 
28 June 1998
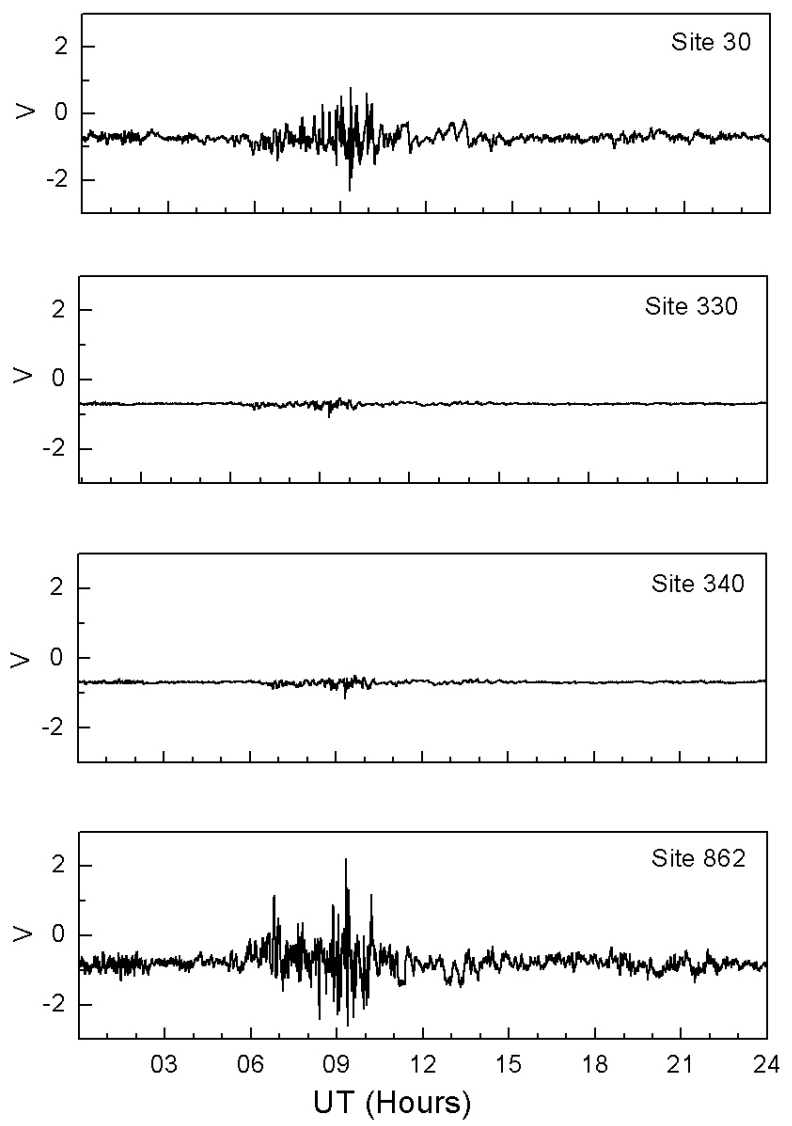

Fig. 10. Modelled pipe-to-soil potential for different sites on the Norman Wells - Zama pipeline for high terminating impedance.

end of the pipeline. There is some uncertainty in the appropriate value to use for the terminating resistances at the ends of the pipeline, due to the presence of devices designed to reduce interference from neighboring installations. Therefore, we have made the model calculations for the two limiting cases of terminating resistances. The PSP variations (Fig. 10), obtained from the model with a high (1000 Ohm) terminating resistance, show large, oppositely-directed, variations at the ends of the pipeline and small variations in the middle, as seen in earlier studies (Boteler and Seager, 1998). Model results with a low $(0.1 \mathrm{Ohm})$ terminating resistance give similar size PSP variations at the four sites (Fig. 11). The two models give the same PSP variations in the middle of the pipeline (note the different scales in Figs. 10 and 11), showing that they are not affected by the value of the terminating resistance, as can also be seen in Fig. 8. In both sets of modelling results, the PSP variations at the sites (30, 330, $340)$ in the northern half are in phase with each other and out of phase with the PSP variations at the site (862) at the southern end of the pipeline.
28 June 1998
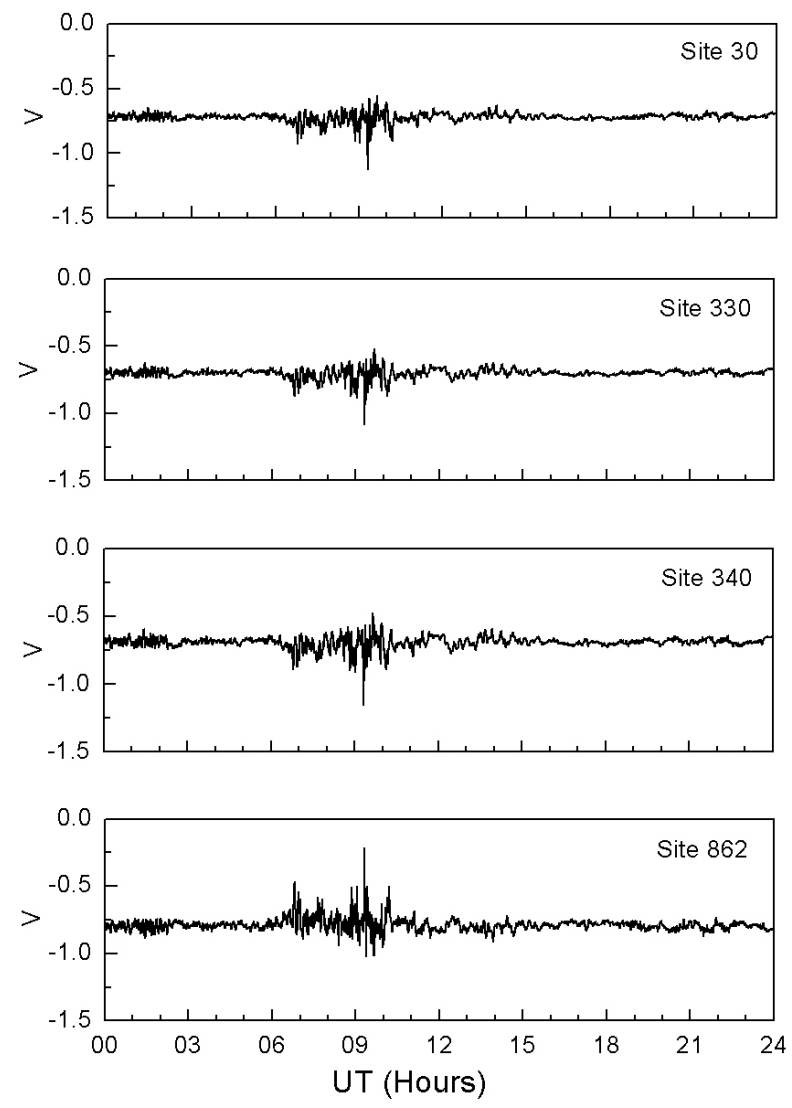

Fig. 11. Modelled pipe-to-soil potential for different sites on the Norman Wells - Zama pipeline for low terminating impedance.

\section{Comparison with observations}

Figure 12 shows recordings of the pipe-to-soil potentials made at the four sites. There is a clear similarity between the pattern of the observed and modelled PSP variations. The observed PSP variations are in phase at sites 30,330, and 340 and out of phase at site 862 . This is in agreement with the modelling results. The relative size of the PSP variations observed at the different sites is in good agreement with the "low terminating resistance" model results, but not with the "high terminating resistance" model results. However, the actual size of the observed PSP variations does not agree with either set of modelling results.

A further comparison of the model and observations was made by plotting the model PSP variations against the observed PSP variations and calculating the linear correlation coefficient (Fig. 13). For sites 330 and 340, both models gave a correlation between the model results and observations of $80 \%$. At site 862 , the "low terminating resistance" model gave a correlation between model results and observations of $70 \%$, with no correlation for the other model. At site 30 , there was no correlation with either model. Sites 330 and 340 , with the highest correlations, are the closest 
28 June 1998
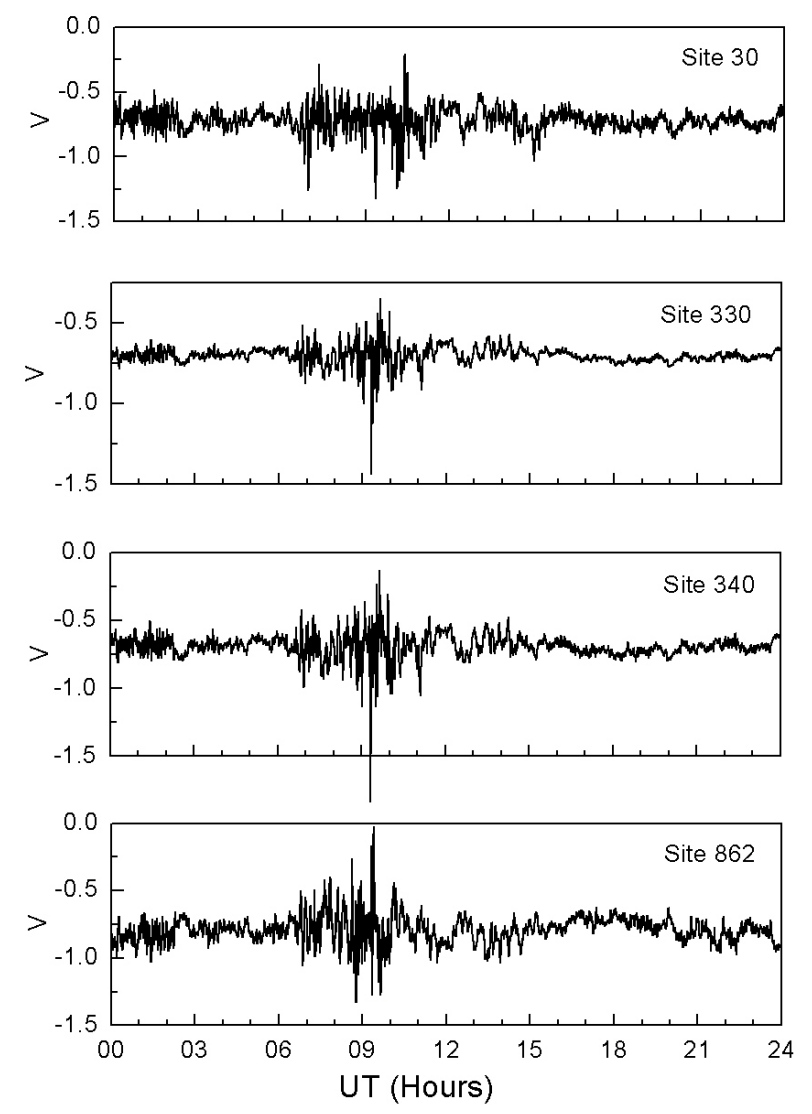

Fig. 12. Observed pipe-to-soil potential for different sites on the Norman Wells - Zama pipeline.

to Fort Simpson, while the sites at increasing distances from Fort Simpson have decreasing values of correlation coefficients. Unfortunately, there was only one day when pipeline measurements and Fort Simpson data were available simultaneously.

Magnetic field variation recordings were also available from Yellowknife magnetic observatory, $370 \mathrm{~km}$ from the pipeline route (Fig. 7). Comparison of the Yellowknife and Fort Simpson magnetic recordings for 28 June shows that the correlation coefficient for the $B_{x}$ component was 0.83 , but for the $B_{y}$ component, it was only 0.40 (no correlation). Comparisons of the electric field calculated for Yellowknife and Fort Simpson magnetic data gave correlation coefficients 0.54 for the $Y$-component and no correlation for the $X$-component. The decrease in correlation shows that the high-frequency part in the electric field increased in comparison with the magnetic field. There was no correlation between observed PSP and modelled with the Yellowknife data.
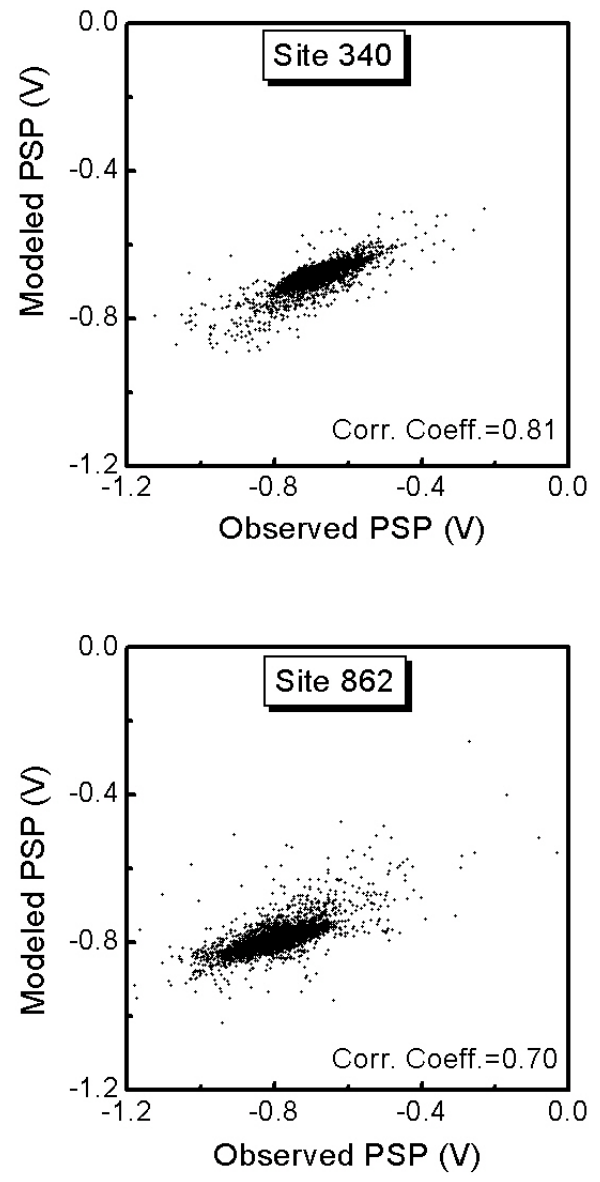

Fig. 13. Linear correlation between modelled and observed pipe-tosoil potential for (a) site 340 and (b) site 862 on the Norman Wells - Zama pipeline.

\section{Discussion}

The model of geomagnetic induction in pipelines presented here gives reasonable results in the time domain appropriate for the comparison with real pipe-to-soil potential measurements. For sites in the middle of the pipeline, the model output and observations are in good agreement, except for a difference in amplitude (model output is $70 \%$ of the observations). Sites at the ends of the pipeline show less correlation. There are a number of features in the modelling that could account for these discrepancies. These are related to two sources: spatial differences in the electromagnetic field on the ground, and spatial differences in the electromagnetic characteristics of the pipeline.

The modelling uses Fort Simpson magnetic data and assumes that this is representative of the magnetic field variations over the whole length of the pipeline. The comparison between the Fort Simpson and Yellowknife magnetic recordings showed a greater correlation in the north- south component of the magnetic field, as would be expected from a predominantly east-west ionospheric current. However, there was a poor correlation for the east-west magnetic field component. The east-west magnetic field variations drive the 
north-south component of the electric field, which is a significant part of the electric field along the pipeline. Thus, we can expect a poor correlation between the electric fields at pipeline sites separated by distances of about $400 \mathrm{~km}$ (the distance between Fort Simpson and Yellowknife).

Spatial variations in the ground conductivity structure will also influence the electric fields along the pipeline route. Some parameters, such as the pipe dimensions and the steel conductivity, are accurately known and unchanging. Possible differences in the Earth's conductivity along different parts of the pipeline were not represented in the one-dimensional layered conductivity structure and will introduce errors in the amplitude of the calculated electric fields. Also, the Earth along the Norman Wells - Zama pipeline route has 2- and 3-dimensional variations in conductivity (Ferguson, private communication, 1999) that can produce localized changes in the electric field strength and direction not represented in the model.

Inaccuracies in the pipeline model would also contribute to disagreements between the model results and observations. Some parameters, such as the pipe dimensions and the steel conductivity are accurately known and unchanging. Other parameters are less well-known, for example, the pipeline coating may have a resistivity value less than the manufacturer's specifications, due to defects introduced during the pipeline construction. As the coating ages there is also a decrease in the resistivity, which would affect the pipe-to-soil potentials. This is illustrated in the design work done for the Maritimes pipelines (Rix and Boteler, 2001).

The pipeline is connected to a refinery at the northern end and another pipeline at the southern end; in both cases there are insulating flanges, but these have degraded over time, thereby allowing some current leakage. Thus, there is considerable uncertainty in the appropriate value to use for the end resistance in the pipeline model. The cathodic protection system on the pipeline uses four main rectifier units plus several smaller units. In normal operation the rectifiers are used in "potential control" mode where they automatically adjust for telluric current fluctuations. For the recording period, the "potential control" was turned off on the main units. The potential control on the smaller units was left on and would have influenced the nearby pipe-to-soil potentials. This may be the reason for the poor correlation obtained at site 30. A test made by comparing only the PSP observations from different sites also shows decreasing correlation with increasing distance between sites (Fig. 14) and indicates that, for the distances larger than $500 \mathrm{~km}$, the assumptions about the plane magnetic field, one dimensional ground conductivity structure and uniform pipeline characteristics were not appropriate.

The DSTL model considered here contains only resistive elements and has a flat frequency response. An improvement would be the inclusion of reactive terms derived from the ILC modelling process. The accuracy of the ILC model is dependent on the input values used, such as the magnetic permeability of the steel and the soil conductivity. The sensitivity

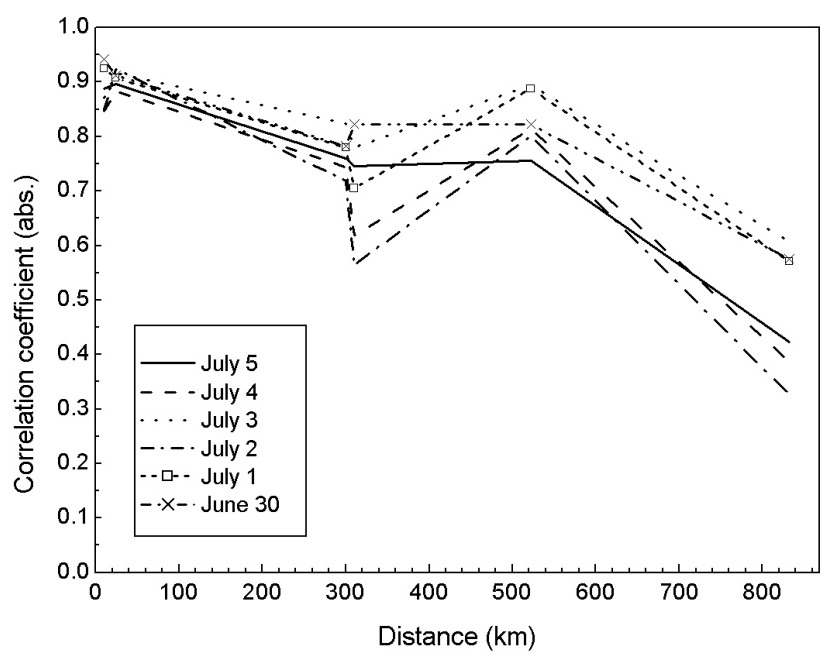

Fig. 14. Correlation between observed PSP at pairs of sites on the Norman Wells - Zama pipeline as a function of the distance between the sites.

of the model to these parameters is examined in Trichtchenko and Boteler (2001).

For the Norman Wells - Zama pipeline, the ILC response is flat over the range of frequencies with which we are concerned, but this may not be the case for a different pipeline or a different frequency range. Increased reactive terms in the DSTL model will generally reduce the size of the pipeto-soil potentials. Inclusion of reactive terms is, therefore, needed if the DSTL modelling is to give accurate results at higher frequencies.

\section{Conclusions}

We have demonstrated the process for modelling geomagnetic effects on pipelines, starting from observed magnetic field variations and including the effect of the Earth's conductivity structure, the reflections from the pipe steel and the pipeline characteristics. Good correlations are obtained between model results and pipeline observations for sites close to a magnetic recording site, but the correlation is poor at distant sites.

The length of the Norman Wells - Zama pipeline $(868 \mathrm{~km})$ is greater than the spatial scales of magnetic disturbances in the auroral zone, and more accurate modelling of the whole pipeline would require magnetic recordings from a number of sites along the pipeline route. Future modelling should also include an improved Earth model to determine how ground conductivity changes along the pipeline route affect the electric fields.

DSTL modelling provides a way of determining the pipeto-soil potentials produced by geoelectric fields. The accuracy of the results is dependent on the quality of the model inputs. The sensitivity of the model results to the end resistances shows the necessity of having detailed information about the electrical characteristics of the pipeline. If higher 
than $0.1 \mathrm{~Hz}$ frequencies are to be considered, then the reactive terms in the pipeline impedance, derived from the ILC modelling, need to be included. With such information, the modelling processes described in this paper could be useful in the design of cathodic protection systems.

Acknowledgement. We are grateful to Bob Lundell for making the recordings of pipe-to-soil potentials. The Fort Simpson magnetometer site is part of the CANOPUS network funded by the Canadian Space Agency. Part of this work was done as part of an international study of telluric current effects on pipelines funded by a consortium of pipeline companies and the Geological Survey of Canada.

The Editor in Chief thanks A. W. P. Thompson and another referee for their help in evaluating this paper.

\section{References}

Boteler, D. H.: Distributed-source transmission line theory for electromagnetic induction studies Proceedings, 1997 Zurich EMC Symposium, Feb. 18-20, URSI supplement, 401-408, 1997.

Boteler, D. H. and Seager, W. H.: Telluric currents: A meeting of theory and observations, Corrosion, 54, 751-755, 1998.

Boteler, D. H. and Trichtchenko, L.: International study of telluric current effects on pipelines, Final Report, Geological Survey of Canada, Open File 3050, 2000.

Boteler, D. H. and Trichtchenko, L.: Observations of telluric currents in Canadian pipelines, Paper 01316, Proceedings CORROSION 2001, NACE, Houston, March 11-16, 2001.

Boteler, D. H., Pirjola, R. J., and Nevanlinna, H.: The effects of geomagnetic disturbances on electrical systems at the Earth's surface, Advances in Space Research, 22, 17-27, 1998.

Campbell W. H.: Induction of the auroral zone electric currents within the Alaska pipeline, Pure and Applied Geophys. 116, 1143-1173, 1978.

Campbell, W. H.: Observation of electric currents in the Alaska oil pipeline Resulting from auroral electrojet current sources. Geophys. J. R. Astr. Soc., 62, 437-449, 1980.

Ferguson, I. J. and Odwar, H. D.: Review of conductivity soundings in Canada, vol 3, Geomagnetic Hazard Assessment, Phase II, Geological Survey of Canada, Open File No 3420, 1997.

Kaufman, A. A. and Keller, G. V.: The magnetotelluric sounding method (Methods in geochemistry and geophysics; 15), 187192, Elsevier Publ. Co., Amsterdam-Oxford-New York, 1981.

Lanzerotti, L. J. and Gregori, G. P.: Telluric currents: The natural environment and interaction with man-made systems, in The Earth's electrical environment, National Academy Press, Washington, D. C., 232-257, 1986.

Peabody, A. W.: Control of pipeline corrosion, National Association of Corrosion Engineers (NACE International), Houston, 1360, 2000.

Place T. D. and Sneath, T. O.: Practical telluric compensation for pipeline close-interval surveys, Materials Performance, 40, 2227, 2001.

Rix, B. C. and Boteler, D. H.: Telluric current considerations in the CP design for the Maritimes and Northeast Pipeline, Paper 01317, Proceedings, CORROSION 2001, NACE, Houston, March 11-16, 2001.

Shapka, R.: Geomagnetic effects on modern pipeline systems, Proceedings, Solar-Terrestrial Predictions Workshop, May 18-22, 1992, Ottawa, 1, 163-170, 1993.

Taflove, A. and Dabkowski, J.: Prediction method for buried pipeline voltages due to $60 \mathrm{~Hz}$ AC inductive coupling, IEEE Trans. Power Apparatus and Systems, vol PAS-98, 780-794, 1979.

Trichtchenko, L. and Boteler, D. H.: Specification of geomagnetically induced electric fields and currents in pipelines, J. Geophys. Res., 106, 21 039-21 048, 2001.

Weast, R. C.: Handbook of Chemistry and Physics, E60-121, CRC Press, Boca Raton, Fla. 1974.

Weaver, J. T.: Mathematical methods for geo-electromagnetic induction, Research Studies Press LTD, Taunton, Somerset, England, 1994. 